\title{
Branching-coalescing particle systems
}

\author{
Siva R. Athreya • Jan M. Swart
}

Received: 15 April 2009 / Revised: 21 May 2009 / Published online: 10 June 2009

(C) Springer-Verlag 2009

\section{Erratum to: Probab. Theory Relat. Fields 131, 376-414 (2005) DOI: $10.1007 / \mathrm{s00440-004-0377-4}$}

It has been pointed out by Martin Hutzenthaler that Theorem 7 in [1] as stated there is incorrect. The purpose of this note is to state a weaker version of the theorem that is still sufficient for our applications in the mentioned paper.

We start by recalling some definitions. If $E$ is a metrizable space, then we denote by $M(E)$ the space of real Borel measurable functions on $E$ and set $B(E):=\{f \in$ $M(E): f$ is bounded\}. If $A$ is a linear operator from a domain $\mathcal{D}(A) \subset M(E)$ into $M(E)$ and $X$ is an $E$-valued process, then we say that $X$ solves the martingale problem for $A$ if $X$ has cadlag sample paths, for each $f \in \mathcal{D}(A)$ one has $E\left[\left|f\left(X_{t}\right)\right|\right]<\infty$ and $\int_{0}^{t} E\left[\left|A f\left(X_{s}\right)\right|\right] \mathrm{d} s<\infty$ for all $t \geq 0$, and the process $\left(M_{t}\right)_{t \geq 0}$ defined by $M_{t}:=f\left(X_{t}\right)-\int_{0}^{t} A f\left(X_{s}\right) \mathrm{d} s(t \geq 0)$ is a martingale with respect to the filtration generated by $X$.

The following theorem corrects [1, Theorem 7].

Theorem 1 (Duality with error term) Assume that $E_{1}, E_{2}$ are metrizable spaces and that for $i=1,2, A_{i}$ is a linear operator from a domain $\mathcal{D}\left(A_{i}\right) \subset B\left(E_{i}\right)$ into $M\left(E_{i}\right)$. Assume that $\Psi \in B\left(E_{1} \times E_{2}\right)$ satisfies $\Psi\left(\cdot, x_{2}\right) \in \mathcal{D}\left(A_{1}\right)$ and $\Psi\left(x_{1}, \cdot\right) \in \mathcal{D}\left(A_{2}\right)$ for each $x_{1} \in E_{1}$ and $x_{2} \in E_{2}$, and that

$$
\Phi_{1}\left(x_{1}, x_{2}\right):=A_{1} \Psi\left(\cdot, x_{2}\right)\left(x_{1}\right) \text { and } \Phi_{2}\left(x_{1}, x_{2}\right):=A_{2} \Psi\left(x_{1}, \cdot\right)\left(x_{2}\right)
$$

The online version of the original article can be found under doi:10.1007/s00440-004-0377-4.

S. R. Athreya

Indian Statistical Institute, 8th mile Mysore Road, RV College PO, Bangalore 560059, India

e-mail: athreya@isibang.ac.in

\section{J. M. Swart $(\varangle)$}

Institute of Information Theory and Automation of the ASCR (ÚTIA),

Pod vodárenskou věží 4, 18208 Praha 8, Czech Republic

e-mail: swart@utia.cas.cz 
$\left(x_{1} \in E_{1}, x_{2} \in E_{2}\right)$ are jointly measurable in $x_{1}$ and $x_{2}$. Assume that $X^{1}$ and $X^{2}$ are independent solutions to the martingale problems for $A_{1}$ and $A_{2}$, respectively, and that

$$
\int_{0}^{T} \mathrm{~d} s \int_{0}^{T} \mathrm{~d} t E\left[\left|\Phi_{i}\left(X_{s}^{1}, X_{t}^{2}\right)\right|\right]<\infty \quad(T \geq 0, i=1,2) .
$$

Then

$$
\begin{aligned}
E & {\left[\Psi\left(X_{T}^{1}, X_{0}^{2}\right)\right]-E\left[\Psi\left(X_{0}^{1}, X_{T}^{2}\right)\right] } \\
& =\int_{0}^{T} \mathrm{~d} t\left(\Phi_{1}\left(X_{t}^{1}, X_{T-t}^{2}\right)-\Phi_{2}\left(X_{t}^{1}, X_{T-t}^{2}\right)\right)
\end{aligned}
$$

holds for a.e. $T$ with respect to Lebesgue measure. Moreover, the left-hand side of (3) is continuous in $T$.

This theorem differs in two ways from [1, Theorem 7]. First, the latter does not contain the statement about the continuity of the left-hand side of (3), and second, it is claimed there that (3) holds for every $T \geq 0$ (instead of a.e. $T$ ). This latter claim is wrong in general. For a counterexample demonstrating this as well as a proof of the corrected theorem we refer to [2].

Theorem 7 in [1] is applied at two places in that article: in the proof of Theorem 1, pages 401-403, and in the proof of Proposition 23, pages 404-405. Luckily, in both instances, all that is needed is the following corollary, which still holds.

Corollary 1 (Everywhere inequality) Under the assumptions of Theorem 1, if

$$
A_{1} \Psi\left(\cdot, x_{2}\right)\left(x_{1}\right) \geq A_{2} \Psi\left(x_{1}, \cdot\right)\left(x_{2}\right) \quad\left(x_{1} \in E_{1}, x_{2} \in E_{2}\right),
$$

then

$$
E\left[\Psi\left(X_{T}^{1}, X_{0}^{2}\right)\right] \geq E\left[\Psi\left(X_{0}^{1}, X_{T}^{2}\right)\right](T \geq 0)
$$

The same statement holds with both inequality signs reversed.

Proof Set $g(T):=E\left[\Psi\left(X_{T}^{1}, X_{0}^{2}\right)\right]-E\left[\Psi\left(X_{0}^{1}, X_{T}^{2}\right)\right]$. Then Theorem 1 shows that $g$ is a continuous function satisfying $g \geq 0$ a.e., hence $g(T) \geq 0$ for every $T \geq 0$.

\section{References}

1. Athreya, S.R., Swart, J.M.: Branching-coalescing particle systems. Prob. Theory Relat. Fields. 131(3), 376-414 (2005)

2. Athreya, S.R., Swart, J.M.: Correction to: Branching-coalescing particle systems. ArXiv:0904.2288v1 